\title{
Three-Dimensional Analysis of Melanosomes Isolated from B16 Melanoma Cells by Using Ultra High Voltage Electron Microscopy
}

\author{
Shuuichi Akazaki ${ }^{*}$, Toshie Takahashi ${ }^{1}$, Yujiro Nakano ${ }^{1}$, Tomoki Nishida ${ }^{2}$, Hirotarou Mori ${ }^{2}$, \\ Akio Takaoka ${ }^{2}$, Hitomi Aoki ${ }^{3}$, Huayua Chen ${ }^{4}$, Takahiro Kunisada ${ }^{3}$, Kenzo Koike ${ }^{1}$ \\ ${ }^{1}$ Beauty Research Center, Kao Corporation, Tokyo, Japan; ${ }^{2}$ Research Center for Ultra-High Voltage Electron Microscopy, Osaka \\ University, Suita, Japan; ${ }^{3}$ Department of Tissue and Organ Development Regeneration, Gifu University, Gifu, Japan; ${ }^{4}$ Department \\ of Anatomy, Gifu University, Gifu, Japan. \\ Email: ${ }^{*}$ akazaki.shuuichi@kao.co.jp
}

Received November $17^{\text {th }}, 2013$; revised December $18^{\text {th }}$, 2013; accepted January $4^{\text {th }}$, 2014

Copyright (C) 2014 Shuuichi Akazaki et al. This is an open access article distributed under the Creative Commons Attribution License, which permits unrestricted use, distribution, and reproduction in any medium, provided the original work is properly cited. In accordance of the Creative Commons Attribution License all Copyrights (C) 2014 are reserved for SCIRP and the owner of the intellectual property Shuuichi Akazaki et al. All Copyright (C) 2014 are guarded by law and by SCIRP as a guardian.

\section{ABSTRACT}

Melanosomes, isolated by centrifugal separation from culture broth of B16 melanoma cells derived from mouse, were observed by scanning electron microscopy (SEM), and by transmission electron microscopy (TEM). Some interesting structural features were found inside and outside of the melanosomes. By SEM observation, the melanosomes were ellipsoid shape, their surface was not smooth and was covered with rough substructure, 10 to 20 nm particles. By TEM, uneven structure and micro particles were observed in the melanosomes. Furthermore, three-dimensional analysis was tried by using the ultra-high voltage electron microscopy (UHVEM). Micrographs of the melanosomes were taken at various tilted angles by UHVEM, after preparing $500 \mathrm{~nm}$ thickness specimens stained with lead citrate. From the micrographs collected, the three- dimensional structures were reconstructed by using i-mode software. Melanin stained by lead and non stained parts was clearly observed in the reconstructed structure. Non stained parts were round, regular size, and distributed widely in the melanosomes.

\section{KEYWORDS}

B16 Melanoma; Melanosome; Scanning Electron Microscopy; Transmission Electron Microscopy; Ultra High Voltage Electron Microscopy; Three-Dimensional Structure

\section{Introduction}

Melanosomes are specialized organelles in pigment cells called melanocyte controlling melanin biosynthesis. The matured melanin is transferred from a pigment cell to neighboring cells, such as keratinocytes. Melanocytes are neural crest-derived cells that exist in the substantia nigra of brain, uveal tract including iris of eye, cochlear duct of ear and basal layer/hair follicle of skin. Melanin contributes to the determination of hair and skin color, the biological defense to ultraviolet rays, etc. in humans.

${ }^{*}$ Corresponding author.
As the surface of the melanosome is central to its functions [1,2], elucidation of the pigment composition of the surface is necessary. Some reports previously have described the three-dimensional ultrastructure of melanosomes [1,3-5]. It is thought that each melanosome consists of structural proteins such as melanin, enzymes related to melanization and carrier proteins of melanin [6]. Melanin can be classified into dark black eumelanin and light red pheomelanin. Melanosome which synthesize predominantly eumelanin are called eumelanosomes, while those that synthesize predominatly pheomelanin are called pheomelanosome. Surface substructure, amino acid contents, metal composition and PTCA/PDCA com- 
position in eumelanosomes/pheomelanosomes and enzymatic/acid/ base were reported [4,5].

Since the molecular structure of melanin polymer was not clear, the detailed inside ultrastructure of melanosome was also still unidentified. In particular, it is unknown where biosynthesis of melanin is performed inside of melanosome. Controlling biosynthesis pathway of melanin is an important theme in the dermatological field and the beauty field. It is thought that the understanding of the inside ultrastructure may lead to the elucidation of the melanization mechanism.

We have examined the internal and external structure of melanosomes. For this purpose, we have used ultrahigh voltage electron microscopy imaging (Osaka Univ.) besides normal transmission electron microscopy imaging. This huge electron microscope makes it possible to obtain a three-dimensional ultrastructure profile $[7,8]$. Reports about inside ultrastructure of melanosome using this three-dimensional electron microscopy have not been reported.

In our first attempt we focused on B16 melanoma cells (B16) derived from mouse.

As a part of investigating the characteristics of melanosomes themselves and their role in controlling the pigment system, we purified melanosomes and successively examined their ultrastructure. B16 cells were cultured, homogenized and melanosome containing fraction was isolated by density difference centrifugation method. We demonstrate characteristic features about the ultrastructural imaging of extracted and isolated melanosomes.

\section{Materials and Methods}

\subsection{Regents}

DMEM; Dulbecco's modified Eagle's medium (GIBCO). FBS; Fetal bovine serum (Equitech-Bio).

EDTA; Ethylenediaminetetraacetic acid (Sigma).

Trypsin (Sigma)

Penicillin (GIBCO).

PBS; Ca-Mg-free phosphate buffer saline (Sigma).

HEPES; N-2-hydroxyethylpiperazine-N-2-ethane sulfonic acid (life technologies).

\subsection{Strains and Culture Condition}

The strain of B16 melanoma derived from mouse, kept as a stock strain at the department of tissue and organ development regeneration of Gifu University, Japan, was used in these experiments. The culture condition of B16 melanoma cells was referred to the Riken's protocol (http://www2.brc.riken.jp/lab/cell/detail.cgi?cell_no=RC B0557\&type $=1$ ). The culture of B16 melanoma cells was performed in a $10-\mathrm{cm}$ dish containing DMEM supple- mented with $10 \%$ FBS and penicillin by using $\mathrm{CO}_{2}$ incubator (ASTEC, Japan) at $37^{\circ} \mathrm{C}$. The cultured melanoma cells which formed a layer on the bottom of the dish could be recovered by addition of drops of $0.25 \%$ trypsin, $1 \mathrm{mM}$ EDTA in $0.7 \times$ PBS. Same DMEM medium successively was added.

For the subculture, the cell suspension was diluted 3 to 6 times of the original concentration with the same DMEM medium. After several times of subcultures, melanoma cell layers on the bottoms are recovered as cell suspension by the same procedure as above. Medium was removed and collected by decantation and washed once by $1 \times$ PBS. Melanoma cells were treated with drops of a solution containing $0.25 \%$ trypsin, $1 \mathrm{mM}$ EDTA in $0.7 \times$ PBS for removal of the cells from the dish.

The subcultured dishes finally increased from 30 to 50 dishes, before the following melanosome preparation step.

\subsection{Preparation 1 of Melanosome}

The melanoma cell suspension was centrifuged for $5 \mathrm{~min}$. at $700 \times \mathrm{g}, 4^{\circ} \mathrm{C}$ to remove supernatant. The melanoma cell pellet was washed with $0.25 \mathrm{M}$ sucrose in $10 \mathrm{mM}$ HEPES buffer, and was centrifuged for $10 \mathrm{~min}$ at $1000 \times$ g., $4^{\circ} \mathrm{C}$.

Pellets were resuspended in HEPES buffer and homogenized with a glass/glass homogenizer (Dounce Tissue Grinder, WHETON, USA) in $0.25 \mathrm{M}$ sucrose in 10 mM HEPES buffer, on ice using 25 strokes. Homogenates centrifuged $10 \mathrm{~min}$ at $1000 \times \mathrm{g}, 4^{\circ} \mathrm{C}$ to remove pellets containing unbroken cells and nuclei. Supernatants were collected and ultra-centrifuged $45 \mathrm{~min}$ at $20,630 \times \mathrm{g}$, $4^{\circ} \mathrm{C}$.

After centrifugation, pellets including melanosomes were precipitated $[9,10]$. Pellets were suspended twice by distilled water and preserved at $-80^{\circ} \mathrm{C}$, temporary. Finally, these were freeze-drying and sealing in glass tubes following preservation long term.

\subsection{Preparation 2 of Melanosome}

Cultured medium was separately collected and centrifuged for $5 \mathrm{~min}$ at $10,000 \times \mathrm{g}, 4^{\circ} \mathrm{C}$ to precipitate melanosomes released from B16 into its medium. These pellets were suspended twice by distilled water and preserved at $-80^{\circ} \mathrm{C}$. These also were freeze-dried and sealing in glass tubes following preservation.

\subsection{Electron Microscopies}

\subsubsection{Scanning Electron Microscopy (SEM)}

Field Emission-SEM JSM-6330F (JEOL, Japan) was 
used to examine external structures in high vacuum mode. The freeze-drying samples were mounted on aluminum specimen mount using electrical conductive double-sided tape. The mounted freeze-drying samples were coated platinum sputtering using ESC-101 (ELIONIX, Japan) before observation.

\subsubsection{Transmission Electron Microscopy (TEM)}

TEM, JEM-1010 (JEOL, Japan) and H-7100 (HITACHI, Japan) were used to examine internal structures. The freeze-drying samples were embedded with Epoxy resin after fixation and dehydration. Usual thin-sections were stained with double-stain of Uranyl acetate dihydrate/ Lead citrate or with Lead citrate only.

\subsubsection{Ultra-High Voltage Electron Microscopy (UHVEM)}

UHVEM was used to examine three-dimensional internal structures. UHVEM (H-3000, HITACHI, Japan) had 3 million volts for acceleration voltage and was located at Research center for Ultra-High Voltage Electron Microscopy, Osaka University. The freeze-drying samples were fixed with normal Epoxy resin. Thin-sections $1 \mu \mathrm{m}$ thickness, and stained with Lead citrate in 10 min incubation at room temperature.

UHVEM acquired images while tilting thin-sections. Single tilt series were recorded over an angular range of 120 degrees ( -60 to +60 degrees). And 60 TEM images sequentially acquired at 2 degrees intervals. Figure 1 gives the graphic representation of this principal for acquisition of three-dimensional Images [11]. TEM images were recorded using a $4096 \times 4096$ pixel slow scan CCD camera (TVIPS, Germany). These tilt series were aligned by the etomo in the IMOD software package

(http://bio3d.colorado.edu) using the gold particles, and tomograms were generated. Three-dimensional projection images were generated by Image-J

(http://rsbweb.nih.gov/ij/), image-analysis software. These softwares can make animation files (video clip, avi file) of tomograms and projection images.

The software (an auto contour and imodauto, which is a threshold-based algorithm) was generated the information of interface between one material and another material. This can visualize the difference of non-specific staining contrast in electron microscopy sample as internal structure and contents [8].

\section{Results and Discussion}

B16 melanoma cells were cultured and subcultured several times according to the Riken's protocol. The cultured cells were harvested for the following preparation step.
The method for preparation of melanosomes from B16 was adapted from previously described method $[9,10]$. B16 cells were harvested with trypsin/EDTA and collected by centrifugation. After suspension in $0.25 \mathrm{M}$ sucrose in $10 \mathrm{mM}$ HEPES buffer, cells were homogenized with a Dounce glass/glass homogenizer. Unbroken cells and nuclei were removed by centrifugation. The supernatants were collected and ultra-centrifuged $45 \mathrm{~min}$ at $20,630 \times \mathrm{g}, 4^{\circ} \mathrm{C}$.

Harvested black pellets were collected as a suspension of B16 melanosomes in water and freeze-dried (Figure 2(a)). Since the samples after freeze-drying are treated like powder, it is thought that samples looked gray or whitish by diffused reflection (Figure 2(b)). B16 secreted melanosomes fraction (Materials and methods 2.4.) are also observed that same color as isolated B16 melanosomes fraction (Figures 2(c), (d)).

Figure 3 shows SEM micrographs of melanosomes isolated from B16. Almost all particles (melanosomes) are ellipsoidal shape. Diameter of particles is long axis of 300 - 400 nanometers and short axis of 200 - 300 nanometers. Its surface is not smooth and uniform but uneven appearance. Diameter of each uneven substructure is 10 20 nanometers.

Figure 4 shows SEM micrographs of melanosomes released (secreted) naturally from B16 to cultured medium. The size and surface structure of released melanosomes are same range and same shape as isolated one with homogenized procedure. About its shape, there are many round shapes in released melanosomes compared with isolated melanosomes.

B16 melanoma cells naturally secrete mature melanosomes to outside of cells. The cultured melanoma cells release melanosome to own medium. The features of their shape and surface substructure were nearly same between isolated melanosomes and released melanosomes. Collection of medium was carried out at every subculture. Naturally released melanosomes are regarded as advanced stage IV. It is unknown whether same entire melanosomes release to keratinocyte in vivo. The yield of released melanosomes was very small, and fluctuated by every subculture. Application of stimulative factors (UV irradiation or chemicals) for stimulation of releasing melanosomes is required.

In this report, isolated melanosome containing fraction included amorphous materials around melanosomes, although these amorphous materials were able to be removed by ethanol treatment. These amorphous contaminations are considered to be residual substance consisting nucleus or proteins or unbroken parts of cell, etc.

Figure 5 shows TEM micrographs of melanosomes isolated from B16. Some unstained parts (small white 


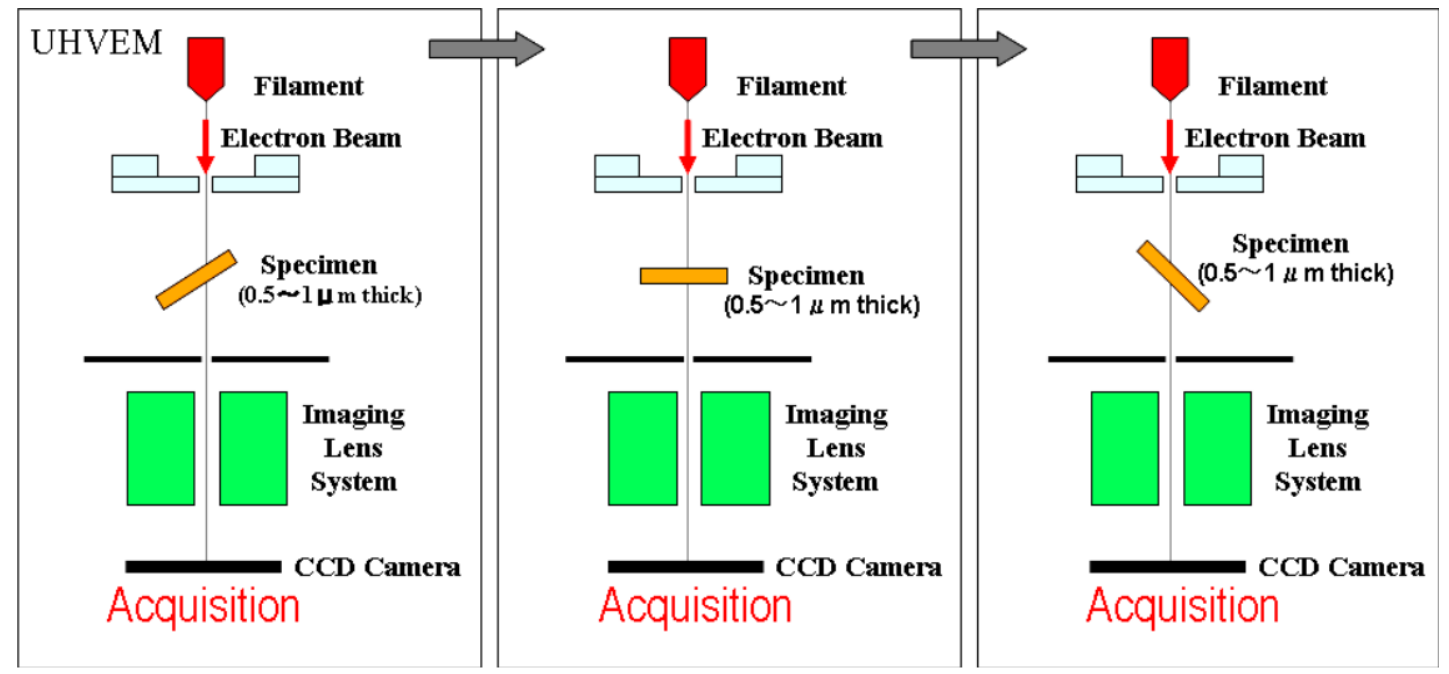

Figure 1. A simplified schema showing the acquisition of sequential images to get three dimensional data by using Ultra-High Voltage Electron Microscopy (UHVEM) at Osaka Univ. Specimen: thin sections for UHVEM. Tilting angles: +60 to -60 degrees. Interval of acquisition: 2 degrees.
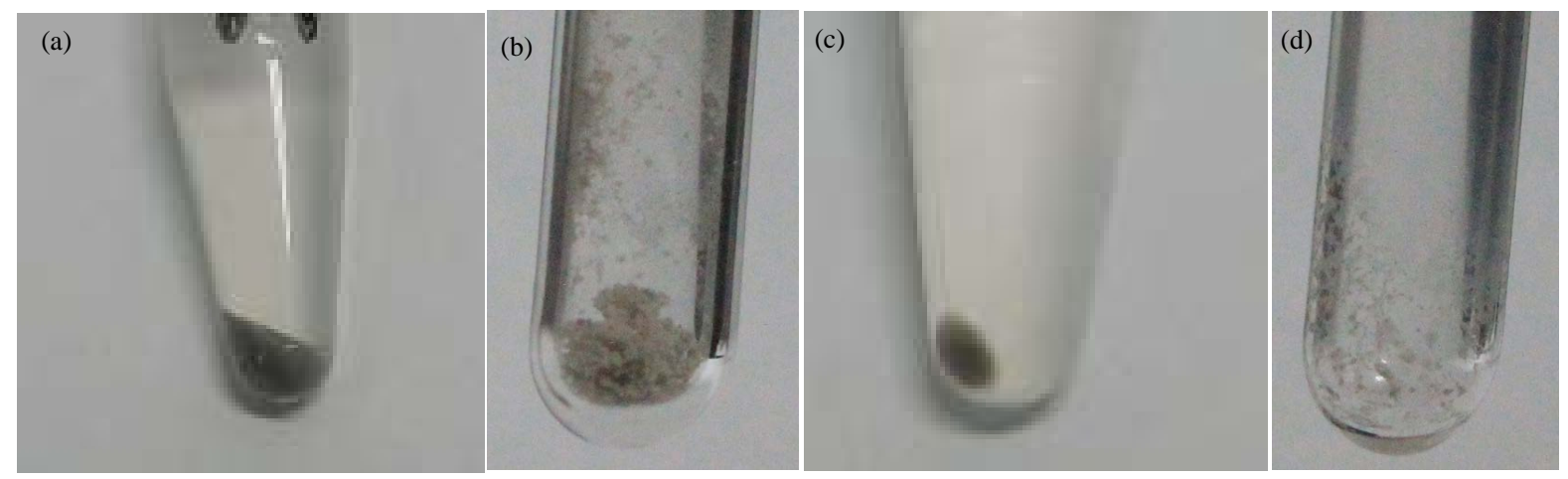

Figure 2. (a) Isolated B16 melanosomes pellets before freeze-drying. (b) Isolated B16 melanosomes samples after freeze- drying in the sealed glass tube. (c) Secreted B16 melanosomes pellets before freeze-drying. (d) Secreted B16 melanosomes samples after freeze-drying in the sealed glass tube.

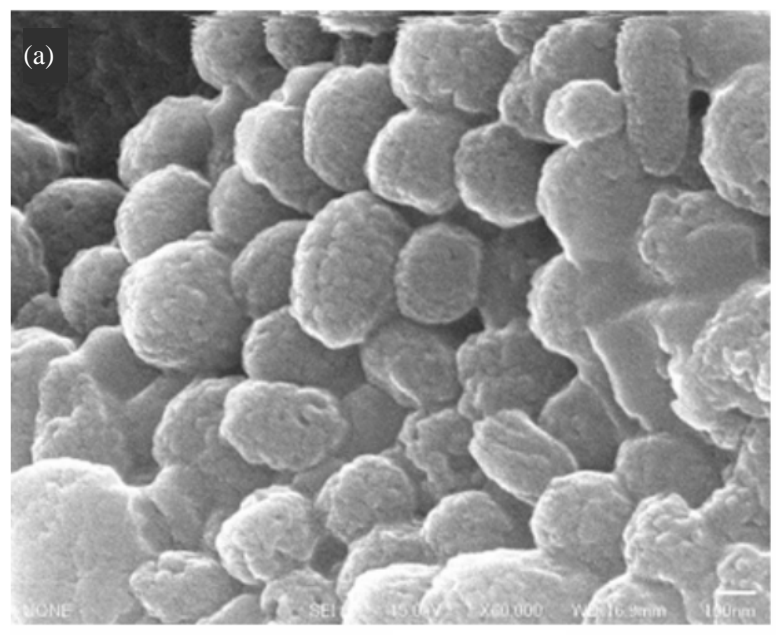

Bar $=100 \mathrm{~nm}$

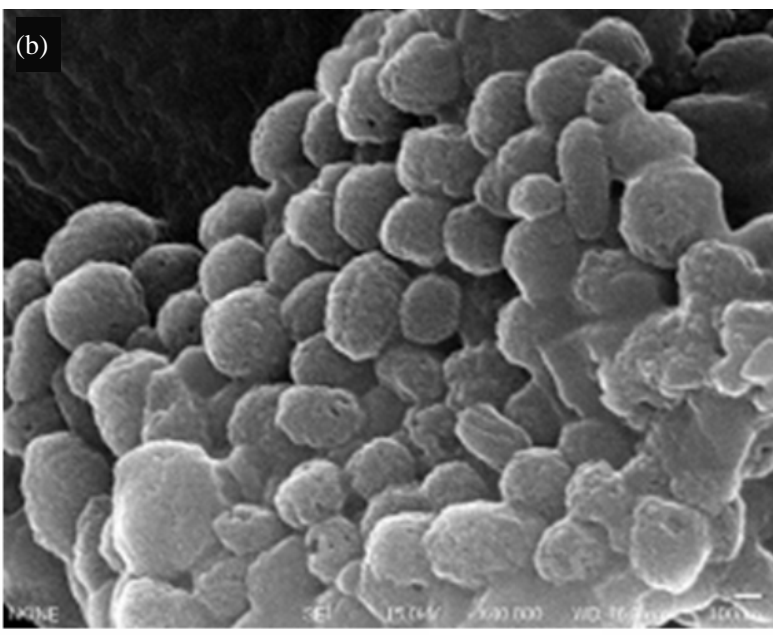

- Bar $=100 \mathrm{~nm}$

Figure 3. (a), (b) SEM micrographs of isolated B16 melanosomes fraction by the preparative method of 2.3. Preparation 1. Bars $=100 \mathrm{~nm}$. 

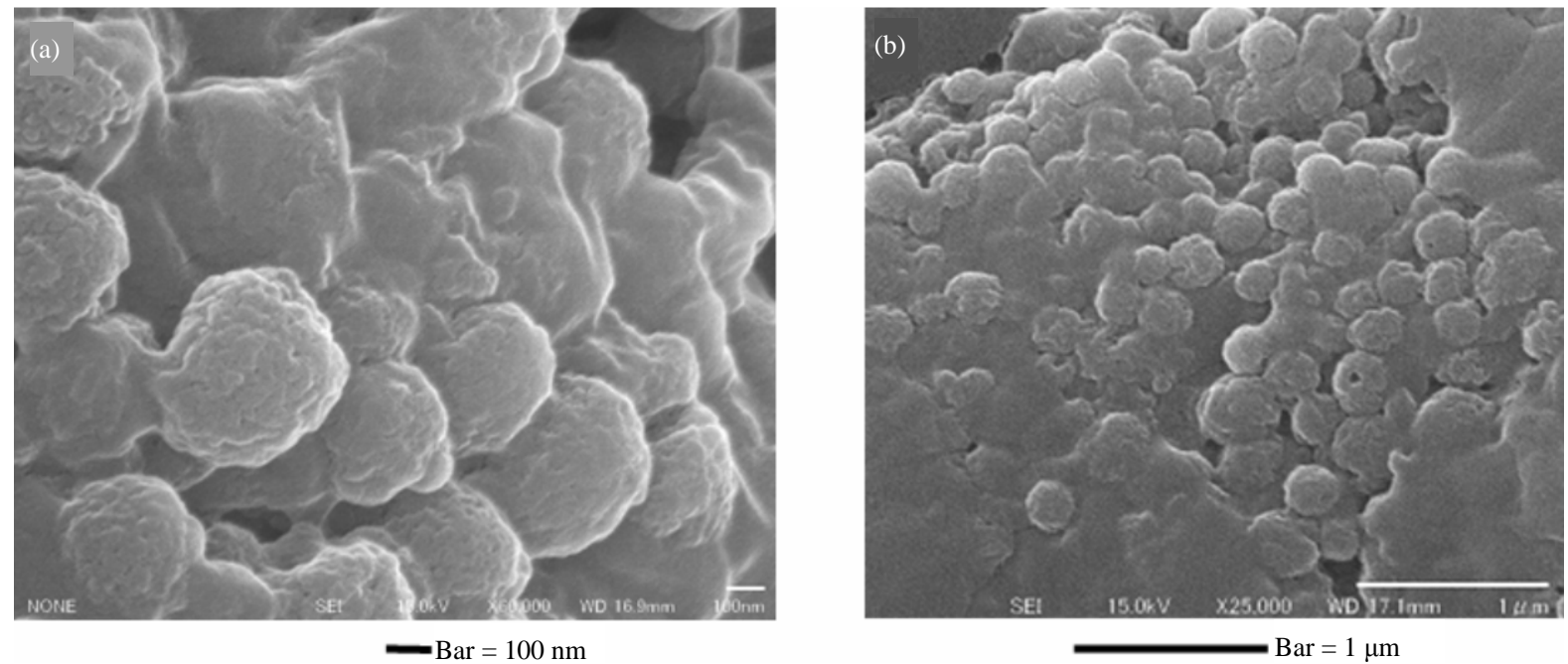

Figure 4. (a), (b) SEM micrographs of B16 secreted melanosomes fraction by the preparative method of 2.4. Preparation 2. Bars: (a) $=100 \mathrm{~nm},(\mathrm{~b})=1 \mu \mathrm{m}$.
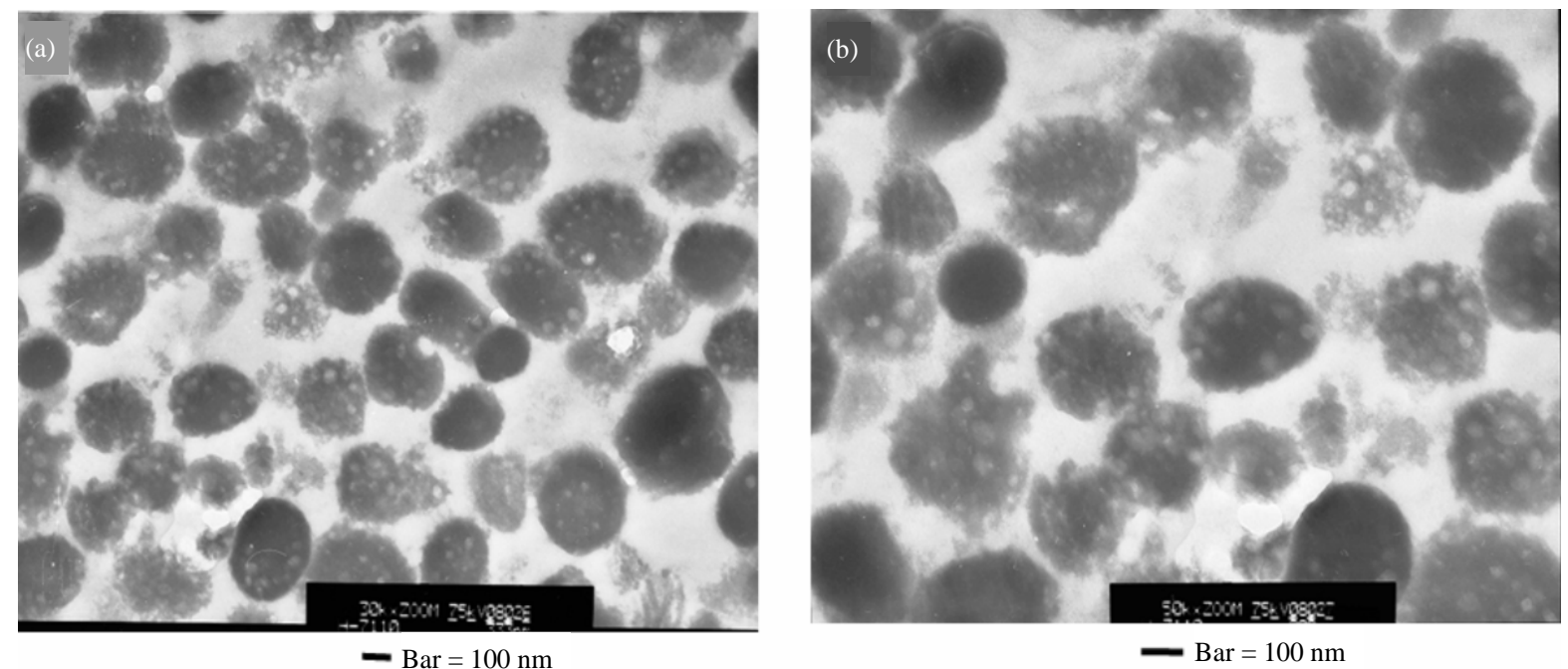

Figure 5. (a), (b) TEM micrographs of isolated B16 melanosomes fraction by the preparative method of 2.3. Preparation 1. Thin sections staining: Lead citrate. Bars $=100 \mathrm{~nm}$.

particles) are observed inside almost all melanosomes. These seem to exist uniformly from the central region to the peripheral region. Size of each unstained part is 10 20 nanometers.

SEM observation would be probing smaller ultrasubstructures along the surface of melanosomes. TEM observation would be probing unstained parts in the melanosomes. As both ultrasubstructure (size of 10 - 20 nanometers and uniform distributions) are comparatively similar, some kind of association may be suggested.

In the observations of TEM and UHVEM, the influence of amorphous contaminations (nucleus or proteins or unbroken parts of cell, etc.) which observed in SEM images was reduced by single stain of lead citrate. Lead citrate stains melanin only.
60 original photographs taken from UHVEM were processed with image-analysis software to generate three-dimensional images. These are outputted as projection animation files (video clip, avi file). Figure 6 shows projection images of melanosomes isolated from B16. These 6 still images ((a)-(f)) were picked out by regular intervals from sequential 60 original projection photographs. 10 to $20 \mathrm{~nm}$ size unstained parts exist widely throughout in melanosomes. Size of these is the same as that of normal TEM images.

Figure 7 shows tomogram (three-dimensional TEM images) of melanosomes isolated from B16. These 6 still images ((a)-(f)) were extracted at regular time intervals from whole tomogram animation (video clip, avi file). Small unstained parts exist every section of tomograms 


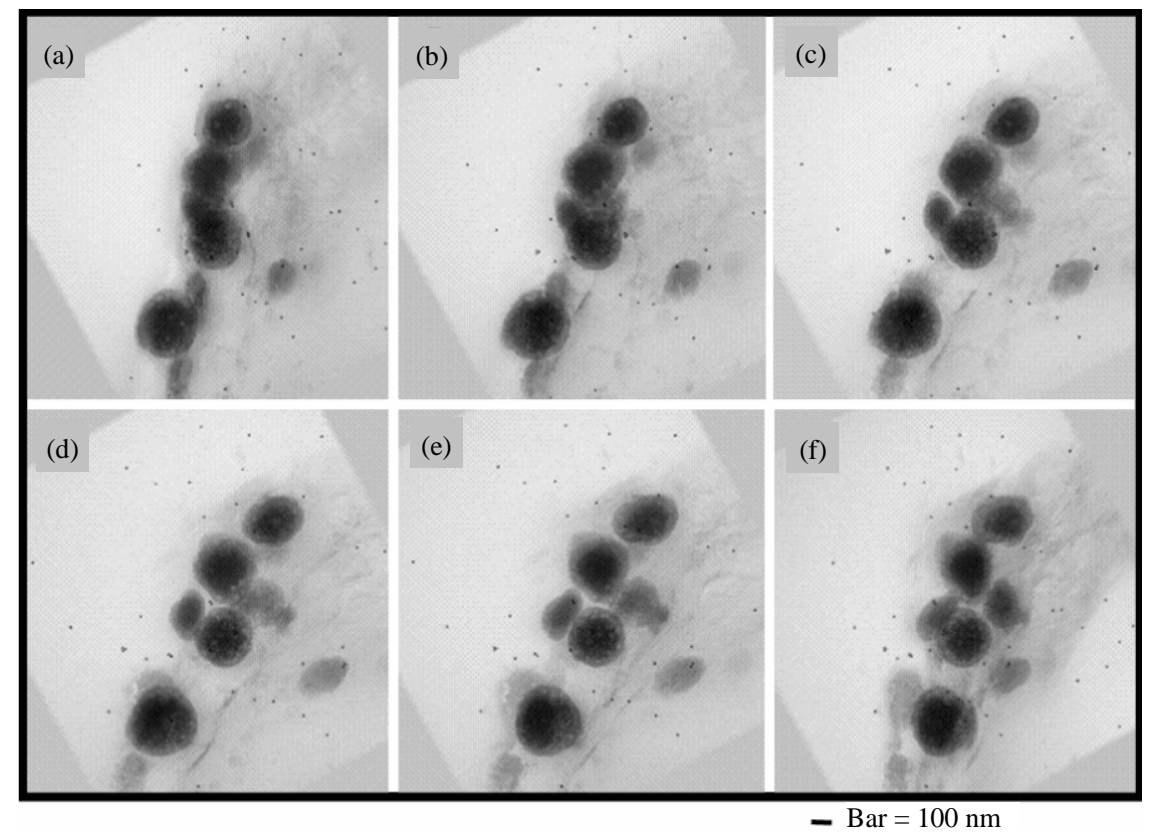

Figure 6. (a)-(f) UHVEM, projection sequential micrographs of isolated B16 melanosomes fraction by the preparative method of 2.3. Preparation 1. Thin sections staining: Lead citrate. These 6 still images were picked out by regular intervals from sequential 60 original projection photographs. Bar $=100 \mathrm{~nm}$.

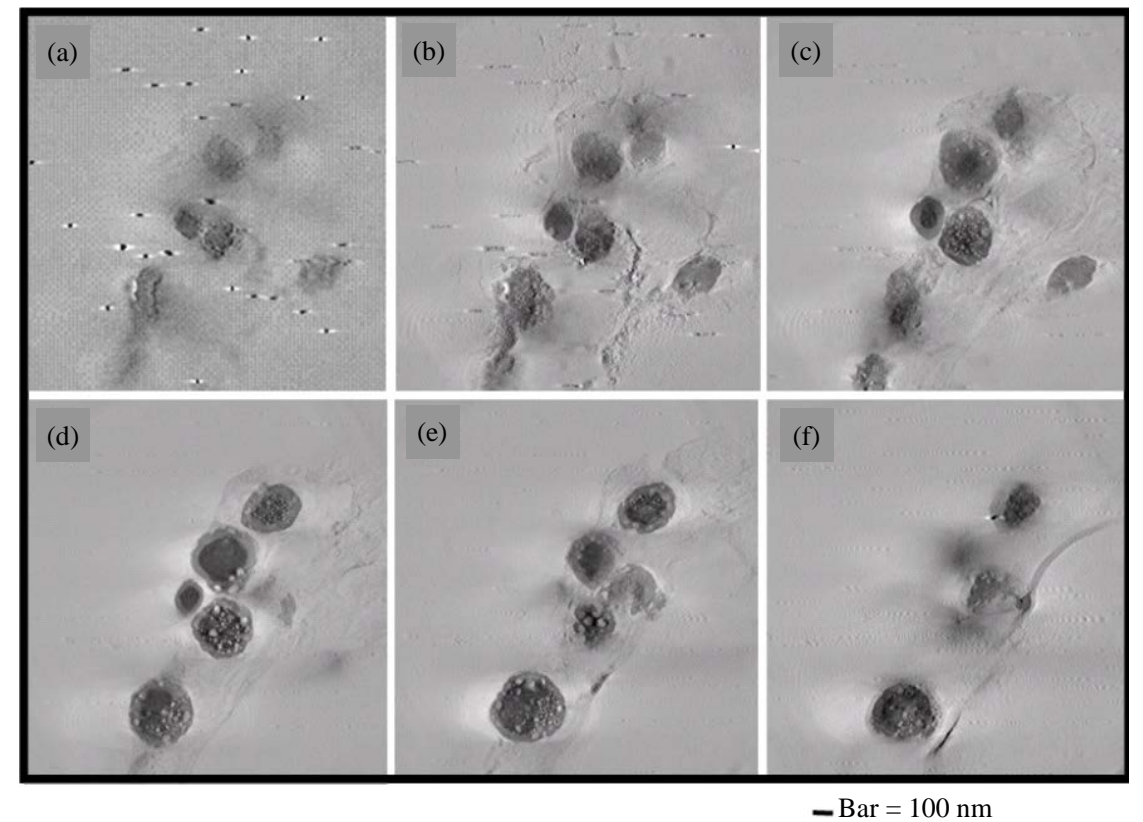

Figure 7. (a)-(f) UHVEM, tomogram sequential images of isolated B16 melanosomes fraction by the preparative method of 2.3. Preparation 1. Thin sections staining: Lead citrate. These 6 still images were extracted by regular time intervals from whole tomogram animation. Bar $=100 \mathrm{~nm}$.

from bottom to top.

Figure 8 shows three dimensional images that indicate the small unstained particles in one melanosome. These 3 still images ((a)-(c)) were extracted at random from whole contour animation. Red lines of coloring by auto contour software correspond to interface between mela- nosome and outside. Green lines of coloring by auto contour software correspond to interface between the unstained particles and another stained part. It is thought that two kinds of substances in melanosomes are different in their physical properties.

Equal and widespread distribution of unstained parts 


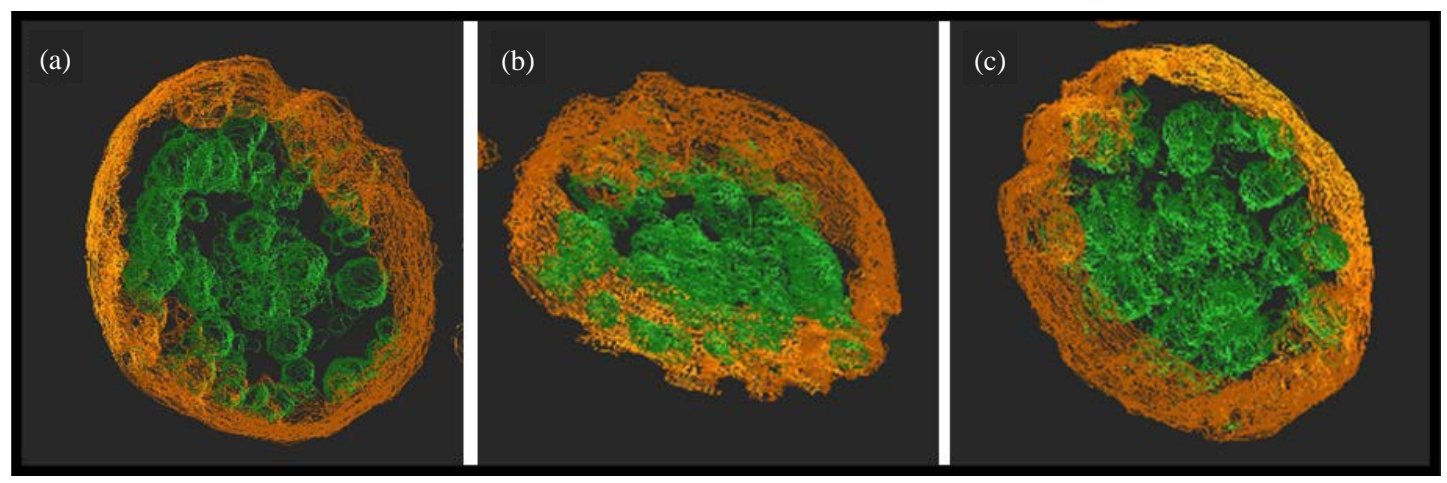

Figure 8. (a)-(c) UHVEM, three dimensional contour images of isolated one B16 melanosome generated using specific software. These 3 still images were extracted at random from whole contour animation.

inside the melanosomes was able to be confirmed by the animation (projection images and tomograms) of UHVEM. There was distributive variation in each melanosome. Some melanosomes had many intrinsic unstained parts. Such variation may be relating mature level of melanosome, difference of stage and/or eumelanin/pheomelanin ratio.

Since the details of the molecular structure of the melanin itself are unknown, little information about threedimensional ultrastructure of melanosomes is available. Some reports previously have been described three-dimensional ultrastructure of melanosomes from iridal stroma, retinal pigment epithelium and human hair [1,3-5].

Free electron laser photoemission electron microscopy data is attributed to eumelanin on the surface of human iridal stroma melanosomes [1]. The surface of the iridal stroma melanosomes is comprised of smaller uneven ultrasubstructures of a few tens of nanometers. Another report shows that eumelanin premelanosomes comprise zigzagging longitudinal strands with cross-links that occur every $20 \mathrm{~nm}$ [12]. From this, they conclude that these surface ultrasubstructures of melanosomes have eumelanin coats. These substructures were found on the surface of the iridal stroma melanosomes all have eumelanin coats [1].

As for a hair, black and red human hairs were analyzed for their structural, chemical and physical properties $[4,5]$. The black-hair melanosomes are ellipsoidal and maintain structural integrity. The red-hair melanosomes are less regularly shaped and are generally smaller than black hair and tend to fall apart. A single black-hair melanosome have packed substructures forming a relatively smooth outer surface. Red-hair melanosomes exhibit a rougher surface, with larger substructures [4]. Eumelanosomes are rod-like and pheomelanosomes are round and more spherical [13]. New born melanosomes are short and round and grow to form rod shaped aging [14].
It is suggested that many B16 melanosomes are eumelanosome type since they are ellipsoidal shape and have small substructures forming uneven outer surface. But there are some more spherical shapes compared with human hair melanosomes which are sharp rod-like. It is necessary to examine ratio of existence of eumelanine and pheomelanine from now on. Recently, chemical analysis has been established [15].

For these examinations, comparative studies of other melanomas or normal melanocytes from various origins may be effective. Melanosomes are classified into four stages according to their maturity. It is said that internal structure of melanosome begins to be formed at stage I, and is completed at stage II. Tyrosinase activity appears and melanization begins at stage, and mature melanosome is completed at stage IV. In this report, it is thought that isolated melanosomes are mature melanosomes because inside melanin of isolated melanosomes was stained and outside structure was nearly the same as released melanosomes. Early stage (I, II) and mature stage (III, IV) of melanosomes could be collected separately using density-gradient centrifugation method [9]. We are considering examining the difference of stages, origins (ex. human races, aging change) about ultrastructure of melanosome.

The constituent of unstained parts has not been analyzed yet. Preliminary analysis by ICP-MS (PerkinElmer, ELAN DRC II ) after acid digestion pretreatment showed the presence of $\mathrm{Cu}$ in melanosome containing fraction sample isolated from B16 (data not shown). Tyrosinase analogues are the rate limiting enzyme binding $\mathrm{Cu}$ for biosynthesis of Dopa. The minimum detectable mole fraction of $\mathrm{Cu}$ ion was detected in retinal pigment epithelium melanosomes of rats by energy dispersive X-ray microanalysis [16]. In human hair, abundant $\mathrm{Cu}$ ion was detected, and the content of black hair was two times higher than red hair [4].

Further analysis should be necessary for localization of 
$\mathrm{Cu}$ or other specific contents in melanosome particle.

\section{Acknowledgements}

This work was supported by Research Center for UltraHigh Voltage Electron Microscopy, Nanotechnology Platform (Microstructural Characterization Platform) in Osaka University of Japan. This work was also supported by Genetic information Genetic Resource Center of Human Pathogens in Gifu University of Japan.

\section{REFERENCES}

[1] D. N. Peles, L Hong, D. N. Hu, S. Ito, R. J. Nemanich and J. D. Simon "Human Iridal Stroma Melanosomes of Varying Pheomelanin Contents Prossess a Common Eumelanic Outer Surface,” Journal of Physical Chemistry B, Vol. 113, No. 32, 2009, pp.11346-11351. http://dx.doi.org/10.1021/jp904138n

[2] J. D. Simon, L. Hong and D. N. Peles "Insights into Melanosomes and Melanin from Some Interesting Spatial and Temporal Properties,” Journal of Physical Chemistry B, Vol. 112, No. 42, 2008, pp. 11201-11217. http://dx.doi.org/10.1021/jp804248h

[3] Y. Liu, L. Hong, K. Wakamatsu, S. lto, B. Adhyaru, C. Y. Cheng, C. R. Bowers and J. D. Simon "Comparison of Structural and Chemical Properties of Melanosomes Isolated from Retinal Pigment Epithelium, Iris and Choroid of Newborn and Mature Bovine Eyes,” Photochemistry and Photobiology, Vol. 81, No. 3, 2005, pp. 510-518. http://dx.doi.org/10.1562/2004-10-19-RA-345.1

[4] Y. Liu, L. Hong, K. Wakamatsu, S. Ito, B. Adhyaru, C. Y. Cheng, C. R. Bowers and J. D. Simon "Comparison of Structural and Chemical Properties of Black and Red Human Hair Melanosomes," Photochemistry and Photobiology, Vol. 81, No. 1, 2005, pp. 135-144. http://dx.doi.org/10.1562/2004-08-03-RA-259.1

[5] Y. Liu, V. R. Kempf, J. B. Nofsinger, E. E. Weinert, M. Rudnicki, K. Wakamatsu, S. Ito and J. D. Simon "Comparison of the Structural and Physical Properties of Human Hair Eumelanin Following Enzymatic or Acid/Base Extraction,” Pigment Cell Research, Vol. 16, No. 4, 2003, pp. 355-365. http://dx.doi.org/10.1034/j.1600-0749.2003.00059.x

[6] Y. Yamaguchi and V. J. Hearing "Physiological Factors That Regulate Skin Pigmentation,” Biofactors, Vol. 35, No. 2, 2009, pp. 193-199. http://dx.doi.org/10.1002/biof.29

[7] K. Koike, A. Mamada, S. Nagase, O. Yoshida and A.
Takaoka "Structure Analysis of Human Hair Fiber with High Voltage Electron Microscopy (HVEM)," Microscopy, Vol. 43, No. 4, 2008, pp. 258-262.

[8] T. Nishida, R. Yoshimura and Y. Endo, "Three- Dimensional Distribution of TrkA Neurotrophin Receptors in Neurite Varicosities of Differentiated PC $_{12}$ Cells Treated with NGF Determined by Immunoelectron Tomography," Cell Tissue Research, Vol. 35, No. 1, 2013, pp. 1-13. http://dx.doi.org/10.1007/s00441-012-1499-0

[9] H. Watabe, T. Kushimoto, J. C. Valencia and V. J. Hearing "Isolation of Melanosomes," Current Protocols in Cell Biology, Vol. 3, 2005, pp. 1-3, 14, 16.

[10] A. Kawase, T. Kushimoto, Y. Kawa, K. Ohsumi, H. Nishikawa, T. Kawakami, M. Mizoguchi and Y. Soma "Proteomic Analysis of Immature Murine Melanocytes at Different Stages of Maturation: A Crucial Role for Calreticulin,” Journal of Dermatological Science, Vol. 49, No. 1, 2008, pp. 43-52. http://dx.doi.org/10.1016/j.jdermsci.2007.07.012

[11] A.Takaoka, T. Hasegawa, K. Yoshida and H. Mori “Microscopic Tomography with Ultra-HVEM and Applications,” Ultramicroscopy, Vol. 108, No. 3, 2008, pp. 230238. http://dx.doi.org/10.1016/j.ultramic.2007.06.008

[12] J. A. Brumbaugh "Ultrastructural Differences between Forming Eumelanin and Pheomelanin as Revealed by the Pink-Eye Mutation in the Fowl," Developmental Biology, Vol. 18, No. 4, 1968, pp. 375-390. http://dx.doi.org/10.1016/0012-1606(68)90047-X

[13] H. Nakagawa and G. Imokawa "Characterization of Melanogenesis in Normal Human Epidermal Melanocytes by Chemical and Ultrastructural Analysis," Pigment Cell Research, Vol. 9, 4, 1996, pp. 175-178. http://dx.doi.org/10.1111/j.1600-0749.1996.tb00106.x

[14] R. D. Grickman, S. L. Jaques, R.T. Hall and N. Kumar "Revisiting the Internal Absorption Coefficient of the Retinal Pigment Epithelium Melanosome," Proceedings of SPIE, Vol. 4257, 2001, pp. 131-141.

[15] S. Ito, Y. Nakanishi, R. K. Valenzuela, M. H. Brilliant, L. Kolbe and K. Wakamatsu, "Usefulness of Alkaline Hydrogen Peroxide Oxidation to Analyze Eumelanine and Pheomelanine in Various Tissue Samples: Application to Chemical Analysis of Human Hair Melanins,” Pigment Cell Melanoma Research, Vol. 24, No. 4, 2011, pp. 605613. http://dx.doi.org/10.1111/j.1755-148X.2011.00864.X

[16] A. Biesemeier, U. Schraermeyer and O. Eibl "Quantitative Chemical Analysis of Ocular Melanosomes in Stained and Non-Stained Tissues,” Micron, Vol. 42, No. 5, 2011, pp. 461-470.

http://dx.doi.org/10.1016/j.micron.2011.01.004 